\title{
Introduction
}

\section{Glioblastoma: an update on pathophysiology and management strategies}

\author{
Mitchel S. Berger, M.D., ${ }^{1}$ Jeffrey N. Bruce, M.D., ${ }^{2}$ \\ Thomas C. Chen, M.D., Ph.D., ${ }^{3}$ \\ and Gelareh Zadeh, M.D., Ph.D., F.R.C.S.C. ${ }^{4}$
}

\begin{abstract}
${ }^{I}$ Department of Neurosurgery, University of California, San Francisco; ${ }^{3}$ Department of Neuro-Oncology, University of Southern California, Los Angeles, California; ${ }^{2}$ Department of Neurosurgery, Columbia University, New York, New York; and ${ }^{4}$ Division of Neurosurgery, University of Toronto, Ontario, Canada
\end{abstract}

Glioblastoma multiforme remains an enigma for neurooncology; our understanding has progressed, but the prognosis is still poor. In this issue of Neurosurgical Focus, the emphasis is on presenting current understanding of the pathophysiology of the disease and the management strategies that stem from it. The issue can be divided into current management strategies and future treatments based on recent advances in understanding glioblastoma pathophysiology, including cancer stem cells, angiogenesis, and tumor heterogeneity.

The first part of the issue is devoted to an evaluation of treatment methods available to patients today. From an operative standpoint, Hawasli et al. present a timely article on the use of stereotactic laser ablation of high-grade gliomas, a procedure that is available to most neurosurgeons using the current armamentarium of imaging and ablative equipment. Lescher and colleagues assert that the time frame for the evaluation of postoperative changes versus residual tumor is important in determining the extent of resection, given increasing data showing survival benefits with radiographically gross- or near-total resections. From a medical perspective, Barbagallo et al. report data on long-term treatment with temozolomide, whereas Pompili et al. describe palliative care and end of life issues for patients with glioblastoma.

The second part of this issue emphasizes the role of future treatments that will stem from recent advances in understanding the pathophysiology of glioblastoma multiforme. Sundar et al. present a timely review on the role of cancer stem cells in glioblastoma, which is followed by Khan and Ehtesham's article on treatment based on targeting glioma stem cells. The importance and role of angiogenesis in glioblastoma pathophysiology is demonstrated in Womeldorff et al.'s article on the role of hypoxia-inducible factor-1, hypoxia, and cytokine-induced angiogenesis. Understanding the role of angiogenesis leads to a better evaluation of the role of bevacizumab in glioblastoma treatment (Castro and Aghi) and the role of MET activation as a reaction to its use (Awad et al.). Tumor heterogeneity and the microenvironment are well recognized in glioblastoma, but recent advances in molecular genomics enable not only a precise diagnosis, but also prognostic implications. Aum et al. present a precise overview of the molecular and cellular heterogeneity found in glioblastoma. Golden et al. report preclinical data on the role of chloroquine and autophagy in glioblastoma. Two genes with important prognostic implications have recently been identified: $I D H$ (Agnihotri et al.) and WT1 (Broaddus et al.). Identification of these targets can lead to new molecularly targeted therapies (Lau et al.).

Treatment of glioblastoma requires a multidisciplinary approach based on understanding the pathophysiology of the disease, which will eventually lead to a better prognosis and quality of life for our patients.

(http://thejns.org/doi/abs/10.3171/2014.9.FOCUS14677)

\section{Disclosure}

The authors report no conflict of interest. 